On Sizing and Shifting the BFGS Update within the Sized Broyden Family of Secant Updates*

\author{
Hiroshi Yabe \\ Hector J. Martinez \\ Richard A. Tapia \\ December, 1993 \\ (revised August 1996)
}

TR9:3-58

*Previously titled "On Sizing Secant Updates from the Broyden Family." 



\title{
On Sizing and Shifting the BFGS Update within the Sized Broyden Family of Secant Updates
}

\author{
H. Yabe, ${ }^{\dagger}$ H.J. Martínez, ${ }^{\ddagger}$ and R.A. TAPIA ${ }^{\S}$
}

August 23, 1996

\begin{abstract}
Recently Contreras and Tapia demonstrated that selectively sizing the BFGS secant update has numerical value. A conclusion of the current study is that there is numerical value in following a sizing of the BFGS update with a shifting of the BFGS update. Our motivation for such a strategy comes from the known fact that the BFGS update is prone to producing updates with large eigenvalues. Hence, sizing may contribute to near singularity, and shifting can help compensate for this deficiency. We follow Contreras and Tapia in our form of selective sizing. Our form of shifting can be viewerl as switching from the BFGS update to an alternative member of the Broyden farnily of updates. Our shift is obtained by minimizing a weighted form of the Byrd-Nocedal measure over a sized Broyden fainily of secant updates. Numerically, our most effective shift is the one that gives the member of the class which is closest to steepest descent in the Byrd-Nocedal measure.
\end{abstract}

\section{Introduction}

We consider quasi-Newton methods for the unconstrained optimization problem

$$
\min _{x \in \mathcal{R}^{n}} f(x)
$$

where $f$ is twice continuously differentiable. Given $x$, an approximation to a minimizer $x_{*}$, to compute $x_{+}$, a new approximation to $x_{*}$, these methods use a local quadratic model of the form

$$
f(x+s) \approx f(x)+\nabla f(x)^{T} s+\frac{1}{2} s^{T} B s
$$

*Research sponsored by NSF Cooperative Agreementt. No. CCR-88-09615 and DOE Grant DEFG0586-ER25017.

${ }^{\dagger}$ Associate Professor, Faculty of Engineering, Science University of Tokyo, 1-3, Kagurazaka, Shinjukuku, Tokyo, Japan <YABE@JPNSUT20.BITNET@pucc.PRINCETON.EDU>

$\ddagger$ Professor, Departamento de Matemáticas, Universidad del Valle, A.A. 25360, Cali, Colombia, and Visiting Scholar, Department of Computational \&. Applied Mathematics and Center for Research in Parallel Computation (CRPC) Rice University, Houston, Texas 77251-1892. Partially supported by COLCIENCIAS CO: 1106-05-307-93<hector@hypatia.univalle.edu.co>

${ }^{5}$ Professor, Department of Computational \& Applied Mathematics and Center for Research in Parallel Computation (CRPC), Rice University, Houston, TX 77251-1892<rat@caam.rice.edu> 
where $B$ is the current approximation to $\nabla^{2} f(x)$, the Hessian of $f$ at $x$, and $\nabla f(x)$ is the gradient of $f$ at $x$. Our particular interest is in secant methods, i.e., methods that have the property that the subsequent Hessian approximation $B_{+}$satisfies the secant equation

$$
B_{+} s=y \equiv \nabla f\left(x_{+}\right)-\nabla f(x) .
$$

A well-known class of such approximations is the Broyden family, and it is generally accepted that the BFGS method is computationally the best method among these secant methods. However, the BFGS update may be unsatisfactory when $B$ has very large eigenvalues. In order to overcome this difficulty, two remedies have been considered. The first is sizing (multiplying the approximate Hessian by an appropriate scalar before it is updated), and the second is switching (updating the approximate Hessian by a different member of the Broyden class).

In this paper, we combine both remedies in two different ways. Thus we consider two types of sized Broyden family updates and propose several sizing strategies as a safeguard for the BFGS method. We present considerable numerical experimentation in an effort to determine if one particular approach is more effective than the others, and if any one of our approaches compares favorably with the numerically successful selective sizing recently suggested by Contreras and Tapia[7]. Our experimentation implies that one approach is superior to the others and this approach is also superior to the Contreras-Tapia BFGS centered Oren-Luenberger approach.

In the remainder of this section, we introduce our notation and present some history concerning sizing and switching. In Section 2, we collect our main tools and discuss some of their properties. In Sections 3 and 4, we derive our sizing strategies. In Section 5, we summarize and collect all formulas used in our new sizing strategies. Our numerical results are presented in Section 6. Finally, in Section 7 we give some concluding remarks.

Following Al-Baali[1], we consider the map

$$
B F G S(X, s, y)=X-\frac{X s s^{T} X}{s^{T} X s}+\frac{y y^{T}}{y^{T} s} .
$$

In terms of this map, the BFGS update of $B$ can be written

$$
\begin{aligned}
B_{+} & =B F(\boldsymbol{s} S(B, s, y) \\
& =B-\frac{B s s^{T} B}{s^{T} B s}+\frac{y y^{T}}{y^{T} s},
\end{aligned}
$$

and the Broyden family of updates of $B$ can be written

$$
\begin{aligned}
B_{+} & =B F\left(r S\left(B+\phi v v^{T}, s, y\right)\right. \\
& =B-\frac{B s s^{T} B}{s^{T} B s}+\frac{y y^{T}}{y^{T} s}+\phi v v^{T},
\end{aligned}
$$

where $\phi$ is a scalar parameter and

$$
v=\sqrt{s^{T} B s}\left(\frac{y}{y^{T} s}-\frac{B s}{s^{T} B s}\right) .
$$


The equality in (1.6) is immediately transparent if one observes that $v^{T} s=0$. Two important subclasses of this family are the convex class, $\phi \in[0,1]$, and the preconvex class, $\phi<0$. The BFGS update corresponds to the choice $\phi=0$, and the DFP update corresponds to the choice $\phi=1$.

Sufficient conditions for $B_{+}$, obtained from the Broyden family given by (1.6), to be symmetric and positive definite are that $B$ is symmetric and positive definite, $y^{T} s>0$, and $\phi>\phi^{\#}$, where

$$
\phi^{\#}=-\frac{1}{\tau-1} \quad \text { with } \quad \tau=\frac{\left(s^{T} B s\right)\left(y^{T} B^{-1} y\right)}{\left(y^{T} s\right)^{2}} .
$$

Note that $\tau \geq 1$, and if $s$ and $y$ are linearly independent, then $\tau>1$.

In 1974, Oren and Luenberger [17] proposed a class of secant methods which they referred to as self-scaling variable metric methods (SSVM). They modified the convex class and obtained, for $\phi \in[0,1]$,

$$
\begin{aligned}
B_{+} & =\gamma\left(B-\frac{B s s^{T} B}{s^{T} B s}+\phi v v^{T}\right)+\frac{y y^{T}}{y^{T} s} \\
& =B F G S\left(\gamma\left(B+\phi v v^{T}\right), s, y\right) \\
& =B F G S(\gamma B, s, y)+\gamma \phi v v^{T} .
\end{aligned}
$$

For fixed $\phi$ the parameter $\gamma$ was to be chosen so that $\kappa_{2}\left(Q^{-1 / 2} B_{+} Q^{-1 / 2}\right) \leq \kappa_{2}\left(Q^{-1 / 2} B Q^{-1 / 2}\right)$, where $\kappa_{2}(A)$ is the $l_{2}$-norm condition number of $A$, and the matrix $Q$ is the symmetric positive definite Hessian of $f$ (which is constant because they assumed $f$ to be quadratic). They suggested two choices for the parameter $\gamma$

$$
\gamma_{O L}=\frac{y^{T} s}{s^{T} B s} \quad \text { and } \quad \gamma_{I O L}=\frac{y^{T} B^{-1} y}{y^{T} s} .
$$

Soon after in 1976, Oren and Spedicato [18] used the $\ell_{2}$-norm condition number, proposed earlier by Davidon [8], to derive optimal parameters $\phi^{*}$ and $\gamma^{*}$ for the sized Broyden family (1.9). Since these choices were in some sense optimal, they intended them to be used at each iteration. However, in 1978 Shanno and Phua [21] claimed that choosing $\phi^{*}$ and $\gamma^{*}$ fresh at each iteration did not perform well and recommended always using the BFGS update $(\phi=0)$ and sizing only at the first iteration. They suggested sizing with $\gamma_{O L}$ given in (1.10).

In 1981, Dennis, Gay, and Welsch [11] proposed their NL2SOL algorithm for the nonlinear least-squares problem. Their basic algorithm was a Gauss-Newton, trust-region method. However, in order to handle large residual problems, they maintained a structured BFGS secant approximation to the second-order part of the least-squares Hessian, and adaptively decided when to use this approximation. A major concern was that since secant methods do not generate Hessian approximations that become arbitrarily accurate as the iteration proceeds, the approximation to the second-order information may impede the fast convergence for zero-residual problems. They observed that $\gamma_{O L}$ in $(1.10)$ had the property that the interval spectrum of $\gamma_{O L} B$ overlaps the interval spectrum of $\nabla^{2} f(x+\theta s)$ for some $\theta \in(0,1)$. For this reason they decided to call multiplication by $\gamma_{O L}$ "sizing" instead of scaling as had previously been used. More importantly they realized 
that in their application sizing with $\gamma_{O L}$ would guarantee that their approximation to the second-order information would converge to zero when the second-order information was zero at the solution. In this application it was critical that the sizing was done at every iteration.

Contreras and Tapia[7] proposed a selective sizing teclinique in the framework of the BFGS trust-region method using the centered Oren-Luenberger sizing factor defined as

$$
\gamma_{C O L}=\frac{y_{-}^{T} s_{-} / s_{-}^{T} s_{-}+y^{T} s / s^{T} s}{s_{-}^{T} B s_{-} / s_{-}^{T} s_{-}+s^{T} B s / s^{T} s} .
$$

Essentially, Contreras and Tapia suggested sizing only when $\gamma_{O L}<1$. They felt that $\gamma_{O L}$ sized the BFGS update too much and suggested $\gamma_{C O L}$ because for $\gamma_{O L}<1$, it follows that $\gamma_{O L}<\gamma_{C O L}<1$. Hence, they viewed $\gamma_{C O L}$ as a dampening or softening of $\gamma_{O L}$.

Their numerical experiments showed that without sizing, DFP is vastly inferior to BFGS; however, when selectively sized, DFP is competitive with BFGS. In their experiments, DFP performed best with the Oren-Luenberger factor $\gamma_{O L}$, and BFGS fit well with the centered Oren-Luenberger factor $\gamma_{C O L}$ for medium dimensional problems. They concluded that their selective sizing with factor $\gamma_{C O L}$ was superior to the alternatives of never sizing, sizing only at the first iteration, or sizing at every iteration.

Several other measures have been used to derive similar kinds of self-scaling updates. These scalings are optimal with respect to the measures used to derive them. These measures include

$$
\begin{array}{rlrl}
\psi(A) & =\operatorname{Tr}(A)-\ln [\operatorname{det}(A)] & & \text { Byrd and Nocedal[4] } \\
\omega(A) & =\frac{\operatorname{Tr}(A)}{n[\operatorname{det}(A)]^{1 / n}} & & \text { Dennis and Wolkowicz }[10] \\
\sigma(A) & =\frac{\lambda_{1}(A)}{\operatorname{det}(A)^{1 / n}} & & \text { Wolkowicz }[23] \\
\varphi(A) & =n \ln [n \omega(A)] & & \text { Todd }[22] \\
& =n \ln \operatorname{Tr}(A)-\ln \operatorname{det}(A) &
\end{array}
$$

In (1.14), $\lambda_{1}(A)$ denotes the largest eigenvalue of $\mathrm{A}$.

Dennis and Wolkowicz [10] used the $\omega$-measure (1.13) to give a specific sense in which $\gamma_{O L}$ (see (1.10)) is the optimal sizing factor for the DFP upclate and $\gamma_{I O L}$ (see $(1.10)$ ) is the optimal sizing factor for the BFGS update.

Byrd and Nocedal [4] introduced the $\psi$-measure (1.12) as a tool for analyzing the global behavior of quasi-Newton methods. The $\psi$-measure was a critical ingredient in the elegant global convergence theory established for methods from the convex Broyden class (except DFP) by Byrd, Nocedal, and Yuan [3].

Fletcher [12] studied the $\psi$-measure (1.12) and used it to establish yet another optimality characterization of the BFGS and DFP secant updates. Todd [22], following Fletcher's approach, used the $\varphi$-measure (1.15) to provide an alternative proof of a result of Dennis and Wolkowicz [10].

Byrd, Liu, and Nocedal[5] used the $\psi$-function to study the preconvex class. They claimed that negative values of $\phi$ in (1.6) are desirable. They also gave conditions on $\phi$ that guaranteed superlinear convergence. Moreover, they minimized $\psi\left(D^{-1 / 2} B_{+} D^{-1 / 2}\right)$ 
with respect to $\phi$ (with $\gamma=1$ ) to obtain the optimal parameter $\phi^{*}$. This is analogous to Strategy 1 in this paper if sizing is not used (see Section 3). Byrd et al. used $D=\nabla^{2} f\left(x_{c}\right)$ in their numerical experiments. Clearly, this $D$ is not available in a secant environment. However, since its use led to good numerical results, a certain validity of the theory has been demonstrated.

\section{Preliminaries}

The previous comments lead us to consider obtaining improved secant upclates by embedding the Broyden family of updates in a larger parametric class of secant updates and then selecting the parameters which optimize a "goodness" or "conditioning" measure. Toward this end we now motivate our choice for the measure function and our choice for the parametric class.

Our choice for the measure is the Byrd-Nocedal $\psi$-measure

$$
\psi(A)=\operatorname{Tr}(A)-\ln [\operatorname{det}(A)] .
$$

This measure involves all the eigenvalues of $A$, and not just the smallest and largest as more traditional condition measures do.

The following known properties of $\psi$ will be important in our theory. A proof can be found in Fletcher [12].

Theorem 2.1 The function $\psi$ defued by (2.1) is strictly convex on the set

$$
\Gamma=\left\{M \in R^{n \times n} \mid M \text { is symmetric and positive definite }\right\} \text {. }
$$

Furthermore, $\psi(A)$ is globally and uniquely minimized by $A=I$ over the set $\Gamma$.

We now motivate our parametric family of secant upclates. Historically it has been observed that the DFP update tends to produce updates with small eigenvalues, while the BFGS update tends to produce updates with large eigenvalues. Hence in working with matrices which have been generated by the BFCSS upclate there is a danger that a particular sizing factor may be excessively small and the sized matrix $\gamma B$ may be nearly singular. In order to overcome this difficulty, in addition to sizing, we consider shifting the sized matrix $\gamma B$, that is, we consider the following update

$$
B_{+}=B F G S(\gamma B+M, s, y) \text {. }
$$

In (2.2), $\gamma$ is a sizing factor which makes the large eigenvalues small, and $M$ is a shifting matrix which moves $\gamma B$ away from near singularity. Note that, for any $\gamma$ and $M$, the update $B F G S(\gamma B+M, s, y)$ satisfies the secant condition (1.3). These motivational comments lead us to consider as our parametric family

$$
\begin{aligned}
B_{+} & =B F G S\left(\gamma B+\phi v v^{T}, s, y\right) \\
& =B F G S(\gamma B, s, y)+\phi v v^{T},
\end{aligned}
$$

where $v$ is given by (1.7), and the matrix $\phi v v^{T}$ is chosen as a shifting matrix $M$ in (2.2). 
Note that the new sized Broyden family (2.3) is somewhat different from the OrenLuenberger family (1.9). The essential difference between them is that in (2.3) the sizing and shifting are independent of each other.

For the two sized Broyden families (1.9) and (2.3), we have the following lemma. Its proof follows from the sufficient conditions stated above (see (1.8)) for $B_{+}$in the Broyden family (1.6) to be symmetric and positive definite.

Lemma 2.1 Assume that $B$ is symmetric and positive definite, and $y^{T} s>0$.

1. If $\gamma>0$ and $\phi>\phi^{\#}$, then $B_{+}$given by (1.9) is symmetric and positive definite.

2. If $\gamma>0$ and $\phi>\gamma \phi^{\#}$, then $B_{+}$given by (2.3) is symmetric and positive definite.

Our objective is how to best choose parameters $\gamma$ and $\phi$ in both (1.9) and (2.3). Oren and Spedicato[18] chose the parameters in (1.9) which minimized an upper bound of $\kappa_{2}\left(D^{-1 / 2} B_{+} D^{-1 / 2}\right)$. Here $D=B$ and $\kappa_{2}(A)$ is the $l_{2}$ norm condition number of $A$ proposed by Davidon[8].

We will use the $\psi$-measure (2.1) instead of $\kappa_{2}$ to compute optimal values of $\gamma$ and $\phi$ in (1.9) and (2.3). We consider minimizing $\psi\left(D^{-1 / 2} B_{+} D^{-1 / 2}\right)$ for some symmetric positive definite matrix $D$ and investigate the performance for $D=B, I$, and $\nabla^{2} f\left(x_{c}\right) .^{1}$

From Theorem 2.1 we see that $B_{x}=D$ uniquely minimizes $\psi\left(D^{-\frac{1}{2}} B_{x} D^{-\frac{1}{2}}\right)$ over the class of symmetric and positive definite matrices. Hence, the choice of weighting matrix $D=B$ gives a least-change secant method flavor, $D=I$ gives a gradient method flavor, and $D=\nabla^{2} f(x)$ gives a Newton's method flavor.

We will consider three types of minimization problems applied to both the OrenLuenberger sized Broyden class (1.9) and our sized Broyden class (2.3). Firstly, for a given sizing factor $\gamma$, we find the optimal secant update in the sized classes by minimizing $\psi$ over the appropriate class to determine the optimal value of $\phi$. Secondly, we do the above with the roles of $\gamma$ and $\phi$ interchanged to find an optimal sizing factor $\gamma$ for a fixed member of the Broyden class (i.e. fixed $\phi$ ). Finally, we determine the optimal sized member of each sized Broyden class by minimizing $\psi$ over (1.9) and over (2.3) in both $\gamma$ and $\phi$. Strategy 1 described in Section 3 concerns this activity for the Oren-Luenberger sized Broyden class (1.9). Strategy 2 described in Section 4 concerns this activity for our sized Broyden class (2.3).

The following algebraic properties of the mal) (1.4) will be very useful in the next two sections. Their proofs can be obtained from straightfoward linear algebra manipulations.

\section{Proposition 2.1 For any $n \times n$ matrix $X$,}

1. The secant condition $[B F G S(X, s, y)] s=y$ is satisfied.

2. If $X$ is symmetric, then $B F G S(X, s, y)$ is also symmetric.

3. If $X$ is symmetric and positive definite, and $y^{T} s>0$, then $B F G S(X, s, y)$ is also symmetric and positive definite.

\footnotetext{
${ }^{1}$ The matrix $D^{1 / 2}$ is defined as the non-singular and symmetric matrix such that $\left(D^{1 / 2}\right)^{2}=D$.
} 
4. If $X$ is symmetric,

$$
\operatorname{Tr}[B F G S(X, s, y)]=\operatorname{Tr}(X)-\frac{\|X s\|^{2}}{s^{T} X s}+\frac{\|y\|^{2}}{y^{T} s} .
$$

5. We have

$$
\operatorname{det}[B F G S(X, s, y)]=\operatorname{det}(X) \frac{y^{T} s}{s^{T} X s}
$$

\section{Strategy 1}

In this section, we derive weighted $\psi$-optimal values for $\gamma$ and $\phi$ in the Oren-Luenberger sized Broyden class (1.9). Consider a given symmetric positive definite matrix $D$. We introduce this weight $D$ into our optimization problem by working with the quantities

$$
\widehat{B}_{+}=D^{-1 / 2} B_{+} D^{-1 / 2}, \quad \hat{B}=D^{-1 / 2} B D^{-1 / 2}, \quad \hat{y}=D^{-1 / 2} y \quad \text { and } \hat{s}=D^{1 / 2} s
$$

and then considering the $D$-weighted Byrd-Nocedal measure $\psi\left(\widehat{B}_{+}\right)$.

Observe that since the Byrd-Nocedal measure $\psi$ is uniquely and globally minimized by the identity matrix, there will be a tendency to choose parameters which in some sense force $\widehat{B}_{+}$to approximate the identity. Now, if $\hat{B}_{+} \approx I$, then $B_{+} \approx D$. Hence the choice $D=B$ gives a least change or secant flavor, the choice $D=\nabla^{2} f(x)$ gives a Newton's method flavor, and the choice $D=I$ gives a steepest descent method flavor. We ask the reader to keep these comments in mind throughout the remainder of the paper and in particular in the numerical experiments section. At this point in the journey it is not at all clear which choice of $D$ will win numerically. Indeed this was a significant part of our motivation for including the three choices in the present study.

We have

$$
\begin{aligned}
\hat{B}_{+} & =\gamma\left(\widehat{B}-\frac{\hat{B} \widehat{s} \hat{s}^{T} \hat{B}}{\hat{s}^{T} \hat{B} \hat{s}}+\phi \hat{v} \hat{v}^{T}\right)+\frac{\widehat{y} \widehat{y}^{T}}{\hat{y}^{T} \hat{s}} \\
& =B F G S\left(\gamma\left(\hat{B}+\phi \widehat{v} \widehat{v}^{T}\right), \hat{s}, \hat{y}\right),
\end{aligned}
$$

where

$$
\hat{v}=\sqrt{\hat{s}^{T} \hat{B} \widehat{s}}\left(\frac{\widehat{y}}{\hat{y}^{T} \hat{s}}-\frac{\hat{B} \hat{s}}{\hat{s}^{T} \hat{B} \hat{s}}\right)=D^{-1 / 2} v
$$

and $v$ is given by (1.7). Note that some cuantities are invariant under the scaling (3.1); i.e.

$$
\hat{y}^{T} \widehat{s}=y^{T} s, \quad \hat{s}^{T} \hat{B} \hat{s}=s^{T} B s, \quad \hat{y}^{T} \hat{B}^{-1} \hat{y}=y^{T} B^{-1} y \quad \text { and } \quad \hat{v}^{T} \hat{B}^{-1} \widehat{v}=v^{T} B^{-1} v=\tau-1 .
$$

However others are not invariant, i.e.,

$$
\begin{gathered}
\|\hat{y}\|^{2}=y^{T} D^{-1} y, \quad\|\hat{B} \hat{s}\|^{2}=\left\|D^{-1 / 2} B s\right\|^{2}=s^{T} B D^{-1} B s \\
\|\hat{v}\|^{2}=v^{T} D^{-1} v=\left(s^{T} B s\right)\left(\frac{y^{T} D^{-1} y}{\left(y^{T} s\right)^{2}}-2 \frac{y^{T} D^{-1} B s}{\left(y^{T} s\right)\left(s^{T} B s\right)}+\frac{s^{T} B D^{-1} B s}{\left(s^{T} B s\right)^{2}}\right) .
\end{gathered}
$$


Thus an application of Proposition 2.1 to the expression (3.2) yields

$$
\begin{aligned}
\operatorname{Tr}\left(\hat{B}_{+}\right) & =\gamma\left(\operatorname{Tr}(\hat{B})-\frac{\|\hat{B} \widehat{s}\|^{2}}{\widehat{s}^{T} \hat{B} \widehat{s}}+\phi\|\widehat{v}\|^{2}\right)+\frac{\|\hat{y}\|^{2}}{\hat{y}^{T} \widehat{s}} \\
& =\gamma\left(\operatorname{Tr}(\hat{B})-\frac{s^{T} B D^{-1} B s}{s^{T} B s}+\phi v^{T} D^{-1} v\right)+\frac{y^{T} D^{-1} y}{y^{T} s}
\end{aligned}
$$

and

$$
\begin{aligned}
\operatorname{det}\left(\hat{B}_{+}\right) & =\operatorname{det}\left(B F\left(\dot{B} S\left(\gamma\left(\hat{B}+\phi \hat{v} \widehat{v}^{T}\right), \hat{s}, \hat{y}\right)\right)\right. \\
& =\frac{\gamma^{n}}{\gamma} \operatorname{det}(\hat{B}) \operatorname{det}\left(I+\phi \hat{B}^{-1} \widehat{v} \widehat{v}^{T}\right) \frac{\hat{y}^{T} \widehat{s}}{\widehat{s}^{T} \hat{B} \widehat{s}} \\
& =\gamma^{n-1} \operatorname{det}(\hat{B})\left(\frac{y^{T} s}{s^{T} B s}\right)(1+\phi(\tau-1)),
\end{aligned}
$$

where $\tau$ is defined in (1.8).

We will be interested in minimizing $\psi\left(\hat{B}_{+}\right)$in $\gamma$ or $\dot{\phi}$ or both for a fixed matrix $D$. Hence we must study $\psi\left(\widehat{B}_{+}\right)$. Using the preceding results, we have

$$
\begin{aligned}
\psi\left(\hat{B}_{+}\right)= & \operatorname{Tr}\left(\hat{B}_{+}\right)-\ln \left(\operatorname{det}\left(\hat{B}_{+}\right)\right) \\
= & \gamma\left(\operatorname{Tr}(\hat{B})-\frac{s^{T} B D^{-1} B s}{s^{T} B s}+\phi v^{T} D^{-1} v\right)+\frac{y^{T} D^{-1} y}{y^{T} s} \\
& -(n-1) \ln \gamma-\ln (\operatorname{det}(\hat{B}))-\ln (1+\phi(\tau-1))-\ln \left(\frac{y^{T} s}{s^{T} B s}\right) .
\end{aligned}
$$

Now, we have

$$
\begin{aligned}
\frac{\partial \psi}{\partial \phi} & =\gamma v^{T} D^{-1} v-\frac{\tau-1}{1+\phi(\tau-1)} \\
\frac{\partial \psi}{\partial \gamma} & =\xi+\phi v^{T} D^{-1} v-\frac{n-1}{\gamma}, \\
\frac{\partial^{2} \psi}{\partial \phi^{2}} & =\frac{(\tau-1)^{2}}{(1+\phi(\tau-1))^{2}}>0, \\
\frac{\partial^{2} \psi}{\partial \gamma^{2}} & =\frac{n-1}{\gamma^{2}}>0,
\end{aligned}
$$

and

$$
\frac{\partial^{2} \psi}{\partial \phi \partial \gamma}=\frac{\partial^{2} \psi}{\partial \gamma \partial \phi}=v^{T} D^{-1} v
$$

where

$$
\xi=\operatorname{Tr}(\hat{B})-\frac{s^{T} B D^{-1} B s}{s^{T} B s} .
$$

Since $\operatorname{Tr}(\widehat{B})$ is the sum of the eigenvalues of $D^{-1 / 2} B D^{-1 / 2}$ and the Rayleigh quotient

$$
\frac{s^{T} B D^{-1} B s}{s^{T} B s}=\frac{\left(B^{1 / 2} s\right)^{T} B^{1 / 2} D^{-1} B^{1 / 2}\left(B^{1 / 2} s\right)}{\left(B^{1 / 2} s\right)^{T}\left(B^{1 / 2} s\right)}
$$


lies between the smallest and the largest eigenvalues of $B^{1 / 2} D^{-1} B^{1 / 2}$, or equivalently of $D^{-1 / 2} B D^{-1 / 2},{ }^{2}$ we have

$$
\xi=\operatorname{Tr}(\widehat{B})-\frac{s^{T} B D^{-1} B s}{s^{T} B s}>0 .
$$

In addition to $\phi^{\#}$, we define

$$
\phi^{\# \#}=-\frac{\xi}{v^{T} D^{-1} v} .
$$

Now we can consider the three types of minimization problems mentioned at the end of Section 2.

\section{[Minimization Problem 1]}

$$
\min _{\phi>\phi \#} \psi\left(\widehat{B}_{+}\right) \text {for a given } \gamma>0
$$

Of course, we desire an optimal value $\phi^{*}$ such that $\phi^{*}>\phi^{\#}$, which will be the case. Since $d^{2} \psi / d \phi^{2}>0, \psi$ is a strictly convex function of $\phi$, hence $d \psi / d \phi=0$ yields the unique global solution of Problem (3.6)

$$
\phi^{*}=\frac{1}{\gamma\left(v^{T} D^{-1} v\right)}-\frac{1}{\tau-1}=\frac{1}{\gamma\left(v^{T} D^{-1} v\right)}+\phi^{\#}>\phi^{\#},
$$

where $\tau$ and $\phi^{\#}$ are given in (1.8). The clioice $D=B$ gives

$$
\phi^{*}=\frac{1}{\gamma(\tau-1)}-\frac{1}{\tau-1}=\frac{1-\gamma}{\gamma(\tau-1)} .
$$

Observe that here $\gamma=1$ implies $\phi^{*}=0$. So with no sizing, BFGS is optimal in this case.

Recall that the choice $D=\nabla^{2} f(x)$ with $\gamma=1$ (no sizing) was considered in the numerical experiments of Byrd et al.[5] and good numerical results were reported.

\section{[Minimization Problem 2]}

$$
\min _{\gamma>0} \psi\left(\hat{B}_{+}\right) \text {for a given } \phi\left(>\phi^{\#}\right) .
$$

As before, since $d^{2} \psi / d \gamma^{2}>0, \psi$ is a strictly convex function of $\gamma$, hence $d \psi / d \gamma=0$ yields the unique global solution of Problem (3.9)

$$
\gamma^{*}=\frac{n-1}{\xi+\phi v^{T} D^{-1} v}=\frac{n-1}{v^{T} D^{-1} v\left(\phi-\phi^{\# \#}\right)}
$$

provided $n>1$ and $\phi>\max \left(\phi^{\#}, \phi^{\# \#}\right)$ (to guarantee $\left.\gamma^{*}>0\right)$.

The choice $D=B$ gives

$$
\gamma^{*}=\frac{1}{1+\phi \frac{\tau-1}{n-1}}
$$

\footnotetext{
${ }^{2}$ If $(\lambda, x)$ is an eigenvalue-eigenvector pair of $B^{1 / 2} D^{-1} B^{1 / 2},\left(\lambda, D^{1 / 2} B^{-1 / 2} x\right)$ is an eigenvalueeigenvector pair of $D^{-1 / 2} B D^{-1 / 2}$.
} 
Note that $\phi=0$ (BFGS) implies $\gamma^{*}=1$, so if we use the BFGS, then no sizing is optimal.

\section{[Minimization Problem 3]}

$$
\min _{\phi>\phi \#} \psi\left(\hat{B}_{+}\right)
$$

The necessary conditions $\partial \psi / \partial \phi=0$ and $\partial \psi / \partial \gamma=0$ give the equations

$$
\begin{gathered}
\phi\left(v^{T} D^{-1} v\right)=\frac{1}{\gamma}-\frac{v^{T} D^{-1} v}{\tau-1}, \\
\xi+\phi v^{T} D^{-1} v-\frac{n-1}{\gamma}=0
\end{gathered}
$$

where $\xi$ is given by (3.4). Now, we have

$$
\begin{aligned}
\frac{1}{\gamma^{*}} & =\frac{1}{n-2}\left(\xi-\frac{v^{T} D^{-1} v}{\tau-1}\right) \\
& =\left(\phi^{\#}-\phi^{\# \#}\right) \frac{v^{T} D^{-1} v}{n-2} \\
\phi^{*} & =\frac{\xi}{(n-2)\left(v^{T} D^{-1} v\right)}-\left(\frac{n-1}{n-2}\right) \frac{1}{\tau-1} \\
& =\frac{1}{n-2}\left(\phi^{\#}-\phi^{\#}\right)+\phi^{\#} .
\end{aligned}
$$

For Problem (3.11), we must investigate the positive definiteness of $\nabla^{2} \psi$. Since the Hessian $\nabla^{2} \psi$ is represented by

$$
\nabla^{2} \psi=\left(\begin{array}{cc}
\psi_{\phi \phi} & \psi_{\phi \gamma} \\
\psi_{\gamma \phi} & \psi_{\gamma \gamma}
\end{array}\right)=\left(\begin{array}{cc}
\frac{(\tau-1)^{2}}{(1+\phi(\tau-1))^{2}} & v^{T} D^{-1} v \\
v^{T} D^{-1} v & \frac{n-1}{\gamma^{2}}
\end{array}\right)
$$

and $\psi_{\phi \phi}=(\tau-1)^{2} /(1+\phi(\tau-1))^{2}>0$, a necessary and sufficient condition for $\nabla^{2} \psi$ to be positive definite is

$$
\operatorname{det}\left(\nabla^{2} \psi\right)>0
$$

i.e.

$$
\frac{(\tau-1)^{2}}{(1+\phi(\tau-1))^{2}}\left(\frac{n-1}{\gamma^{2}}\right)-\left(v^{T} D^{-1} v\right)^{2}>0 .
$$

Now it is easy to prove the following lemma.

Lemma 3.1 The pair $\left(\phi^{*}, \gamma^{*}\right)$ given by (3.12)-(3.13) is the unique global solution of Problem (3.11).

Proof: Substituting $\left(\phi^{*}, \gamma^{*}\right)$ into (3.14), we lave

$$
\operatorname{det}\left(\nabla^{2} \psi\left(\phi^{*}, \gamma^{*}\right)\right)=(n-2)\left(v^{T} D^{-1} v\right)^{2}>0
$$


Therefore $\nabla^{2} \psi\left(\phi^{*}, \gamma^{*}\right)$ is positive definite and $\left(\phi^{*}, \gamma^{*}\right)$ is a local solution. The uniqueness follows from the fact that $\left(\phi^{*}, \gamma^{*}\right)$ is the unique solution of the necessary conditions $\partial \psi / \partial \phi=0$ and $\partial \psi / \partial \gamma=0$.

The choice $D=B$ gives $\gamma^{*}=1$ and $\phi^{*}=0$. This implies that the BFGS update without sizing is optimal for this particular choice of weighting matrix.

Summarizing the preceding results, we obtain the following proposition.

\section{Proposition 3.1}

1. The parameter $\phi^{*}$ in Problem (3.6) is greater than $\phi^{\#}$.

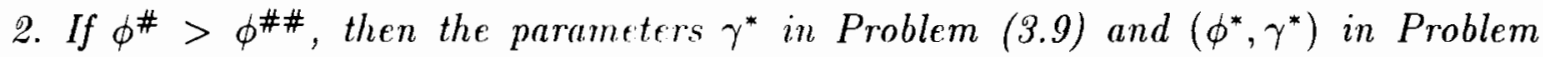
(3.11) satisfy the conditions

$$
\phi^{*}>\phi^{\#} \quad \text { and } \quad \gamma^{*}>0
$$

\section{$4 \quad$ Strategy 2}

In this section, we derive weighted $\psi$-optimal values for $\gamma$ and $\phi$ in our new sized Broyden family (2.3). Recall that this new family is different from the Oren-Luenberger family (1.9). The essential difference between (1.9) and (2.3) is that in (2.3), the matrix $\phi v v^{T}$ is used as a shifting operator and only the matrix $B$ is sized. By doing so; we can separately control the sizing parameter and the shifting term. As before we work with $\psi\left(\widehat{B}_{+}\right)$. We have

$$
\begin{aligned}
\hat{B}_{+} & =B F\left(\hat{s} S\left(\gamma \hat{B}+\phi \hat{v} \widehat{v}^{T}, \widehat{s}, \widehat{y}\right)\right. \\
& =\gamma\left(\hat{B}-\frac{\hat{B} \hat{s}^{T} \hat{B}}{\widehat{s}^{T} \widehat{B} \widehat{s}}\right)+\phi \widehat{v} \widehat{v}^{T}+\frac{\widehat{y} \hat{y}^{T}}{\widehat{y}^{T} \hat{s}}
\end{aligned}
$$

where

$$
\hat{v}=\sqrt{\widehat{\hat{s}^{T} \hat{B} \widehat{s}}}\left(\frac{\hat{y}}{\hat{y}^{T} \hat{s}}-\frac{\widehat{B} \hat{s}}{\hat{s}^{T} \hat{B} \hat{s}}\right) .
$$

Using the same arguments as in Section 3, we have

$$
\begin{aligned}
\operatorname{Tr}\left(\widehat{B}_{+}\right) & =\gamma\left(\operatorname{Tr}(\hat{B})-\frac{\|\hat{B} \hat{s}\|^{2}}{\hat{s}^{T} \hat{B} \widehat{s}}\right)+\phi\|\hat{v}\|^{2}+\frac{\|\hat{y}\|^{2}}{\widehat{y}^{T} \hat{s}} \\
& =\gamma\left(\operatorname{Tr}(\widehat{B})-\frac{s^{T} B D^{-1} B s}{s^{T} B s}\right)+\phi v^{T} D^{-1} v+\frac{y^{T} D^{-1} y}{y^{T} s}
\end{aligned}
$$

and

$$
\begin{aligned}
\operatorname{det}\left(\hat{B}_{+}\right) & =\operatorname{det}\left(B F \operatorname{GS}\left(\gamma \hat{B}+\phi \hat{v} \hat{v}^{T}, \hat{s}, \hat{y}\right)\right) \\
& =\gamma_{n-1} \operatorname{det}(\hat{B})\left(\frac{y^{T} s}{s^{T} B s}\right)\left(1+\frac{\phi}{\gamma}(\tau-1)\right),
\end{aligned}
$$

where $\tau$ is defined in (1.8). 
Thus,

$$
\begin{aligned}
\psi\left(\widehat{B}_{+}\right)= & \operatorname{Tr}\left(\widehat{B}_{+}\right)-\ln \left(\operatorname{det}\left(\widehat{B}_{+}\right)\right) \\
= & \gamma \xi+\phi v^{T} D^{-1} v+\frac{y^{T} D^{-1} y}{y^{T} s} \\
& -(n-2) \ln \gamma-\ln (\operatorname{det}(\widehat{B}))-\ln (\gamma+\phi(\tau-1))-\ln \left(\frac{y^{T} s}{s^{T} B s}\right),
\end{aligned}
$$

where $\xi$ is given by (3.4).

Then we have

$$
\begin{aligned}
\frac{\partial \psi}{\partial \phi} & =v^{T} D^{-1} v-\frac{\tau-1}{\gamma+\phi(\tau-1)} \\
\frac{\partial \psi}{\partial \gamma} & =\xi-\frac{n-2}{\gamma}-\frac{1}{\gamma+\phi(\tau-1)} \\
\frac{\partial^{2} \psi}{\partial \phi^{2}} & =\frac{(\tau-1)^{2}}{(\gamma+\phi(\tau-1))^{2}}>0 \\
\frac{\partial^{2} \psi}{\partial \gamma^{2}} & =\frac{n-2}{\gamma^{2}}+\frac{1}{(\gamma+\phi(\tau-1))^{2}}>0 \\
\text { and } \quad \frac{\partial^{2} \psi}{\partial \phi \partial \gamma} & =\frac{\partial^{2} \psi}{\partial \gamma \partial \phi}=\frac{\tau-1}{(\gamma+\phi(\tau-1))^{2}}>0
\end{aligned}
$$

Now, we can consider the same minimization problems for function (4.2) as we did for function (3.3).

\section{[Minimization Problem 1]}

$$
\min _{\phi>\gamma \phi \#} \psi\left(\widehat{B}_{+}\right) \text {for a given } \gamma(>0) \text {. }
$$

Of course, we need an optimal value $\phi^{*}$ such that $\phi^{*}>\gamma \phi^{\#}$ (see Lemma 2.1), which will be the case. Since $d^{2} \psi / d \phi^{2}>0, \psi$ is a strictly convex function in $\phi$. Hence $d \psi / d \phi=0$ yields the unique global solution of Problem (4.4)

$$
\phi^{*}=\frac{1}{v^{T} D^{-1} v}-\frac{\gamma}{\tau-1}=\frac{1}{v^{T} D^{-1} v}+\gamma \phi^{\#}>\gamma \phi^{\#} .
$$

The choice $D=B$ gives

$$
\phi^{*}=\frac{1-\gamma}{\tau-1}
$$

Note that $\gamma=1$ (no sizing) implies $\phi^{*}=0$ (BFGS).

\section{[Minimization Problem 2]}

$$
\min _{\gamma>0} \psi\left(\hat{B}_{+}\right) \text {for a given } \phi\left(>\gamma \phi^{\#}\right) \text {. }
$$

As before, since $d^{2} \psi / d \gamma^{2}>0, \psi$ is a strictly convex function of $\gamma$, hence the equation $d \psi / d \gamma=0$ implies

$$
\xi-\frac{n-2}{\gamma}-\frac{1}{\gamma+\phi(\tau-1)}=0 .
$$


This yields a quadratic equation of $\gamma$

$$
\xi \gamma^{2}+(\xi \phi(\tau-1)-(n-1)) \gamma-\phi(\tau-1)(n-2)=0 .
$$

Thus we have

$$
\gamma=\frac{1}{2 \xi}[(n-1)-\xi \phi(\tau-1) \pm \sqrt{\delta}]
$$

where

$$
\delta=(\xi \phi(\tau-1)-(n-1))^{2}+4 \xi \phi(\tau-1)(n-2) .
$$

Considering the positivity of $\gamma$, we choose

$$
\gamma^{*}=\frac{1}{2 \xi}[(n-1)-\xi \phi(\tau-1)+\sqrt{\delta}] .
$$

Now we investigate the positivity of $\gamma$. In the choice $\phi>0$, we have

$$
\delta=(\xi \phi(\tau-1)-(n-1))^{2}+4 \xi \phi(\tau-1)(n-2)>0 .
$$

In the choice $\phi \leq 0$, we have

$$
\delta=(\xi \phi(\tau-1)+(n-1))^{2}-4 \xi \phi(\tau-1)>0 .
$$

Then, for any $\phi$,

$$
\gamma^{*}=\gamma^{*}(\phi)=\frac{1}{2 \xi}[(n-1)-\xi \phi(\tau-1)+\sqrt{\delta}>0
$$

so we must choose a parameter $\phi$ such that $\phi>\gamma^{*}(\phi) \phi^{\#}$.

The choice $D=B$ gives $\xi=n-1$; therefore

$$
\gamma^{*}=\frac{1}{2}\left(1-\phi(\tau-1)+\sqrt{(\phi(\tau-1)-1)^{2}+4 \phi(\tau-1) \frac{n-2}{n-1}}\right)
$$

Note that $\phi=0$ implies $\gamma=1$.

\section{[Minimization Problem 3]}

$$
\min _{\phi>\gamma \phi^{*}} \psi\left(\hat{B}_{+}\right)
$$

Since

$$
\frac{\partial^{2} \psi}{\partial \phi^{2}}=\frac{(\tau-1)^{2}}{(\gamma+\phi(\tau-1))^{2}}>0
$$

and

$$
\begin{aligned}
\operatorname{det}\left(\nabla^{2} \psi(\phi, \gamma)\right) & =\frac{(\tau-1)^{2}}{(\gamma+\phi(\tau-1))^{2}}\left(\frac{n-2}{\gamma^{2}}+\frac{1}{(\gamma+\phi(\tau-1))^{2}}\right)-\frac{(\tau-1)^{2}}{(\gamma+\phi(\tau-1))^{4}} \\
& =\frac{(\tau-1)^{2}}{(\gamma+\phi(\tau-1))^{2}}\left(\frac{n-2}{\gamma^{2}}\right)>0
\end{aligned}
$$


the function $\psi\left(\hat{B}_{+}\right)$given by $(4.2)$ is strictly convex with respect to $(\phi, \gamma)$. Thus, for Problem (4.10), a stationary point is the unique optimal point. The equations

$$
\frac{\partial \psi}{\partial \phi}=0 \quad \text { and } \quad \frac{\partial \psi}{\partial \gamma}=0
$$

yield

$$
\begin{gathered}
\frac{1}{\gamma+\phi(\tau-1)}=\frac{v^{T} D^{-1} v}{\tau-1}, \\
\xi-\frac{n-2}{\gamma}-\frac{1}{\gamma+\phi(\tau-1)}=0 .
\end{gathered}
$$

Therefore we obtain the unique global solution for Problem (4.10) .

$$
\begin{aligned}
\gamma^{*} & =\frac{n-2}{\xi-\frac{v^{T} D^{-1} v}{\tau-1}}=\frac{1}{\phi^{\#}-\phi^{\# \#}} \frac{n-2}{v^{T} D^{-1} v}, \\
\phi^{*} & =\frac{1}{v^{T} D^{-1} v}-\frac{\gamma^{*}}{\tau-1}=\frac{1}{v^{T} D^{-1} v}+\gamma^{*} \phi^{\#}>\gamma^{*} \phi^{\#} .
\end{aligned}
$$

The choice $D=B$ gives $\gamma^{*}=1$ and $\phi^{*}=0$. This implies that the BFGS update without sizing is optimal for this choice of weighting matrix.

Summarizing the preceding results, we obtain the following proposition.

\section{Proposition 4.1}

1. The parameter $\phi^{*}$ in Problem (4.4) is greater than $\gamma \phi^{\#}$.

2. The parameter $\gamma^{*}$ in Problem (4.7) is positive for all $\phi$. (Recall that for $B_{+}$to be positive definite, the parameter $\phi$ must bf chosen so that $\phi>\gamma^{*}(\phi) \phi^{\#}$.)

3. If $\phi^{\#}>\phi^{\# \#, ~ t h e n ~ t h e ~ p a r a m e t e r s ~}\left(\phi^{*}, \gamma^{*}\right)$ in Problem (4.10) satisfy the conditions

$$
\phi^{*}>\phi^{\#} \gamma^{*} \quad \text { and } \quad \gamma^{*}>0
$$

\section{Relationships Between Strategies}

In this section, we summarize the main results obtained in Sections 3 and 4 and present some relationships among them. Let the $\psi$-optimal values in Strategies 1 and 2 be $\phi_{1}^{*}, \gamma_{1}^{*}$ and $\phi_{2}^{*}, \gamma_{2}^{*}$, respectively. Assuming that $B$ is symmetric and positive definite, and $y$ and $s$ are linearly independent, and $y^{T} s>0$, the results concerning the three minimization problems we considered are the following.

\section{[Minimization Problem 1]}

Since, for $\gamma$ positive, we have

$$
\phi_{1}^{*}=\frac{1}{\gamma\left(v^{T} D^{-1} v\right)}+\phi^{\#} \text { and } \phi_{2}^{*}=\frac{1}{v^{T} D^{-1} v}+\gamma \phi^{\#}
$$


we obtain the relationship

$$
\phi_{2}^{*}=\gamma \phi_{1}^{*}
$$

Moreover, these optimal values always produce symmetric positive definite updates.

\section{[Minimization Problem 2]}

For a given $\phi$, we have

$$
\gamma_{1}^{*}=\frac{n-1}{\xi+\phi v^{T} D^{-1} v}
$$

and

$$
\gamma_{2}^{*}=\frac{1}{2 \xi}\left[(n-1)-\xi \phi(\tau-1)+\sqrt{(\xi \phi(\tau-1)-(n-1))^{2}+4 \xi \phi(\tau-1)(n-2)}\right],
$$

where

$$
\xi=\operatorname{Tr}\left(D^{-1 / 2} B D^{-1 / 2}\right)-\frac{s^{T} B D^{-1} B s}{s^{T} B s}>0 .
$$

In this case, these optimal values do not always produce a positive definite update. However, $\phi \geq 0$ is sufficient, but not necessary, for these optimal values to produce symmetric positive definite updates.

\section{[Minimization Problem 3]}

Since

$$
\begin{aligned}
\phi_{1}^{*} & =\frac{\xi}{(n-2)\left(v^{T} D^{-1} v\right)}+\left(\frac{n-1}{n-2}\right) \phi^{\#}=\frac{1}{\gamma_{1}^{*} v^{T} D^{-1} v}+\phi^{\#}, \\
\gamma_{1}^{*} & =\frac{n-2}{\xi+v^{T} D^{-1} v \phi^{\#}},
\end{aligned}
$$

and

$$
\begin{aligned}
\phi_{2}^{*} & =\frac{1}{v^{T} D^{-1} v}+\gamma_{2}^{*} \phi^{\#} \\
\gamma_{2}^{*} & =\frac{n-2}{\xi+v^{T} D^{-1} v \phi^{\#}}
\end{aligned}
$$

we obtain the relationships

$$
\phi_{2}^{*}=\gamma^{*} \phi_{1}^{*} \quad \text { and } \quad \gamma^{*} \equiv \gamma_{1}^{*}=\gamma_{2}^{*}
$$

Once again, even though these optimal values do not always produce positive definite updates, $\phi \geq 0$ is sufficient, but not necessary, for these optimal values to produce symmetric positive definite updates.

Finally, observe that, in the choice $D=B$, the standard BFGS update $(\phi=0, \gamma=1)$ is optimal using either Strategy 1 or 2 for Problem 1 with $\gamma=1$, for Problem 2 with $\phi=0$, and for Problem 3. This is certainly an interesting finding and begs for more understanding. 


\section{Numerical Experiments}

Contreras and Tapia[7] obtained impressive numerical results for the BFGS trust-region method using selective sizing and a sizing factor $\gamma_{C O L}$ they called centered Oren-Luenberger. In this section, we present considerable numerical experimentation using various forms of the sizing strategies described in Sections 3 and 4. Our objective, of course, is to determine if one particular approach is more effective than the others, and if any one of our approaches compares favorably with the Contreras-Tapia approach. Hence, we include this latter approach in our numerical studies.

Our sizing strategies were adlapted to both the trust-region and line-search algorithms in an enhanced MATLAB version of the well-known Dennis-Schnabel code[9]. This code uses the double-dogleg implementation of trust-region globalization and the backtracking implementation of line-searcl globalization.

Our experimentation implies that one approach is superior to the others and this approach is also superior to the Contreras-Tapia BFGS approach. The best sizing procedure was derived from our new sized Broyden family (2.3) (Strategy 2) as the solution of Minimization Problem 1 given by (4.4) with $D=I$ and $\gamma=\gamma_{C O L}$, the centered OrenLuenberger sizing introduced by Contreras and Tapia. Later on, we call this approach I2-COL. In fact, Method I2-COL was efficient (see Table 3), robust to parameter changes (see Tables 5 and 6), and reliable as the problem size increased (see Table 7). ${ }^{3}$ See Section 7 for a plausible explanation as to why I2-COL is our most effective strategy.

\subsection{Implementation}

We incorporated into our code the options of shifting and sizing the approximation to the Hessian before it is updated. Thus, before the BFGS updating formula is applied to a matrix $B$, we have the option of modifying $B$ either by

$$
B \leftarrow \gamma\left(B+\phi v v^{T}\right) \quad(\text { Oren-Luenberger sizing (1.9)) }
$$

or by

$$
B \leftarrow \gamma B+\phi v v^{T} \quad(N e w \text { sizing }(2.3)),
$$

depending on whether Strategy 1 (Section 3) or Strategy 2 (Section 4) is being used. Here $\gamma$ and $\phi$ are real scalars and $v \in R^{n}$ is given by (1.7).

We also included in the code the ability to accept an arbitrary initial matrix $B_{0}$. Our choices for the initial matrix $B_{0}$ were

$$
\begin{aligned}
& B_{0}^{0}=\left|f\left(x_{0}\right)\right| I, \\
& B_{0}^{1}=\operatorname{diag}\left(10,10^{4}, 1, \ldots, 1\right), \\
& B_{0}^{2}=\operatorname{diag}\left(10^{-1}, 10^{-4}, 1 \ldots, 1\right), \\
& B_{0}^{3}=\operatorname{diag}(0.1,2,0.5,10,1 \ldots, 1) .
\end{aligned}
$$

In the first expression of $(6.3), x_{0}$ denotes the starting point. Observe that $B_{0}^{0}$ has representative eigenvalues, $B_{0}^{1}$ has large eigenvalues, $B_{0}^{2}$ has small eigenvalues, and $B_{0}^{3}$

\footnotetext{
${ }^{3}$ Other methods were "equally" efficient, but not as robust and reliable. See Methods BFGS-COL, I1-OL, I2-OL for example.
} 
has moderate size eigenvalues. In all of our experiments we allowed a maximum of 500 iterations. The stopping criteria employed is the same as that used in the Dennis-Schnabel optimization code, in particular, convergence is reached when the relative gradient is less than $10^{-7}$. The machine used to obtain these numerical results was a SUN SPARC10/Model 41.

Our test set consists of five problems. Four of them are problems 1, 2, 5 and 21 (with $\mathrm{n}=6$ ) from the standard set of test functions in Moré, Garbow and Hillstrom[14]. The other one is the strictly convex quartic function of Byrd, Liu, and Nocedal[5]. This latter function was also used by Al-Baali[2] in similar numerical testing. For each problem, we chose the standard starting point included in the respective reference.

In (6.1) and (6.2), two values were considered for $\phi: 0$ (BFGS) and $\phi^{*}$ (minimizers given in Sections 3 and 4$),{ }^{4}$ and three values for $\gamma$ : the Oren-Luenbergèr sizing factor $\gamma_{O L}$ given in (1.10), the Centered Oren-Luenberger sizing factor $\gamma_{C O L}$ given by $(1.11)$, and $\gamma^{*}$ (minimizer given in Sections 3 and 4). As suggested by Contreras and Tapia[7], to prevent us from inadvertently sizing with an excessively small constant, the actual sizing factor used was $\max \left\{\gamma_{\min }, \gamma\right\}$, where $\gamma$ is the computed sizing factor, and $\gamma_{\min }$ is a small positive constant.

Our numerical experimentation concerned the following 14 sizing procedures and the standard BFGS method for comparison purposes.

0. BFGS $(\gamma=1, \phi=0$, Standard BF(iS).

1. OL-BFGS $\left(\gamma=\gamma_{O L}, \phi=0\right.$, Standard sized BFGS). ${ }^{5}$

2. ('OL-BFGS $\left(\gamma=\gamma_{C O L}, \phi=0\right.$, Standard sized BFGS) ${ }^{6}$

3. B1-OL $\left(\gamma=\gamma_{O L}, \phi=\phi^{*}\right.$ given by (3.7), Strategy 1, Min. Prob. $\left.1, D=B\right)$.

4. B1-COL $\left(\gamma=\gamma_{C O L}, \phi=\phi^{*}\right.$ given by (3.7), Strategy 1, Min. Prob. $\left.1, D=B\right)$.

5. B2-OL $\left(\gamma=\gamma_{O L}, \phi=\phi^{*}\right.$ given by (4.5), Strategy 2 , Min. Prob. $\left.1, D=B\right)$.

6. B2-COL $\left(\gamma=\gamma_{C O L}, \phi=\phi^{*}\right.$ given by (4.5), Strategy 2, Min. Prob. $\left.1, D=B\right)$.

7. I1-OL $\left(\gamma=\gamma_{O L}, \phi=\phi^{*}\right.$ given by (3.7), Strategy 1, Min. Prob. $\left.1, D=I\right)$.

8. I1-COL $\left(\gamma=\gamma_{C O L}, \phi=\phi^{*}\right.$ given by (3.7), Strategy 1, Min. Prob. 1, $\left.D=I\right)$.

9. I2-OL $\left(\gamma=\gamma_{O L}, \phi=\phi^{*}\right.$ given by (4.5), Strategy 2, Minimization Problem $\left.1, D=I\right)$.

10. I2-COL $\left(\gamma=\gamma_{C O L}, \phi=\phi^{*}\right.$ given by (4.5), Strategy 2, Min. Prob. $\left.1, D=I\right)$.

11. $112^{*}\left(\gamma=\gamma^{*}\right.$ given by $(3.10), \phi=0$, Strategy 1 , Minimization Problem $\left.2, D=I\right)$.

12. I22* $\left(\gamma=\gamma^{*}\right.$ given by (4.8), $\phi=0$, Strategy 2, Min. Prob. $\left.2, D=I\right)$.

13. I13* $\left(\gamma=\gamma^{*}, \phi=\phi^{*}\right.$ given by (3.12) and (3.13), Strategy 1, Minimization Problem $3, D=I)$.

\footnotetext{
${ }^{4}$ Since not always $\phi^{*}>\phi^{\#}$ (or $\phi^{*}>\gamma \phi^{\#}$ ), $\phi^{*}$ was used only if $\phi^{*}>0.95 \phi^{\#}$ (or $\phi^{*}>0.95 \gamma \phi^{\#}$ ).

${ }^{5}$ Oren-Luenberger-sized BFGS, Oren[16], and Oren and Luenberger[17].

${ }^{6}$ Centered Oren-Luenberger sized BFGS, Contreras and Tapia[7].
} 
14. I23* $\left(\gamma=\gamma^{*}, \phi=\phi^{*}\right.$ given by (4.11) and (4.12), Strategy 2, Minimization Problem $3, D=I)$.

\subsection{Preliminary Results}

An important observation from our initial experimentation concerns how often sizing should be performed. If used at every iteration, the sized secant strategies suggested in Sections 3 and 4 were inferior to the standard BFGS in all problems except the last one. ${ }^{7}$ Therefore, sizing should be used as a saffeguarding of the BFGS secant method; in other words, we size or shift $B$ before it is updated only when a need is detected (Selective Sizing/Shifting). It is interesting that this is what has been concluded by numerous authors including Carter[6], Contreras and Tapia[7], Al-Baali[1],[2], and Wolkowicz[23]. Following Contreras and Tapia[7], to decide when to size, we use the fact that both $\gamma_{O L}$ and $\gamma_{C O L}$ should be "close" to 1 whenever $B$ has "good" information relative to $\nabla^{2} f(x)$ along the direction $s$. We consider the following two Selective Sizing/Shifting criteria:

1. Selective Sizing/Shifting Criterion 1

For $k=1,2, \ldots$. if $1-\bar{\gamma}>\epsilon$ then size or shift $B$.

2. Selective Sizing/Shifting Criterion 2

For $k=1,2, \ldots$

if $|1-\bar{\gamma}|>\epsilon$ then size or shift $B$.

We should mention that our preliminary experimentation included other choices of $\phi$ such as 1 (DFP) and other choices of $\gamma$ such as $\gamma_{I O L}=y^{T} B^{-1} y / y^{T}$ s (inverse OrenLuenberger[17]). The results of these experiments showed that selectively sizing the approximation matrix $B$ using $\gamma^{*}$ from Sections 3 or 4 as the sizing factor (see Minimization Problem 2 in Sections 3 and 4) improved the beliavior of DFP much more than it improved the behavior of BFGS. However, these sizing strategies never made DFP competitive with the standard BFGS. This concurs with the common belief that BFGS is a more robust update than DFP, and therefore, DFP is more likely to be improved by a safeguarding technique such as sizing. We believe that further tuning of these sizing procedures is needed in order to obtain the competitive behavior of DFP reported by Contreras and Tapia[7]. Since no better results than those of the standard BFGS method are expected for the sized DFP method, we excluded it from further numerical experiments.

These results also showed that the choice of $\gamma_{I O L}$ gave similar results to the choice of $\gamma_{O L}$. This is not surprising since both quantities contain essentially the same information concerning $B$ relative to $\nabla^{2} f(x)$. For this reason, and because it takes $O\left(n^{2}\right)$ more operations to compute, we excluded $\gamma_{I O L}$ from further numerical experiments.

\subsection{Results}

\footnotetext{
${ }^{7}$ For this particular problem suggested by Byrd et.al.[5], sizing or shifting the matrix in the ways proposed above did improve the behavior of BFGS. This agrees with the results reported by Byrd et.al.[5] and by Al-Baali[2] using a special member of the Broyden Class different from BFGS (shifting).
} 
Table 1: Normalized Averages, $\left(1-\gamma_{O L}>0.05, \quad \gamma_{m i n}=0.1\right)$

\begin{tabular}{|l|c|c|c|c|c|c|}
\hline \multirow{2}{*}{ METHOD } & \multicolumn{3}{|c|}{ LINE SEARCH } & \multicolumn{3}{c|}{ TRUST REGION } \\
\cline { 2 - 7 } & Iterations & Functions & Fun.-Grad. & Iterations & Functions & Fun.-Grad. \\
\hline 0. BFGS & 1.0000 & 1.0000 & 1.0000 & 1.0000 & 1.0000 & 1.0000 \\
1. OL - BFGS & 0.8659 & 0.9677 & 0.8662 & 0.9533 & 1.0392 & 0.9538 \\
2. COL-BFGS & 0.9340 & 0.9848 & 0.9337 & 1.0041 & 1.1300 & 1.0038 \\
3. B1 - OL & 0.9139 & 0.9777 & 0.9141 & 0.9551 & 0.9971 & 0.9555 \\
4. B1 - COL & 0.9248 & 0.9663 & 0.9250 & 0.9681 & 1.0115 & 0.9684 \\
5. B2 - OL & 0.9138 & 0.9747 & 0.9141 & 0.9592 & 1.0048 & 0.9596 \\
6. B2 - COL & 0.9285 & 0.9709 & 0.9287 & 0.9589 & 1.0056 & 0.9593 \\
7. I1 - OL & $\mathbf{0 . 8 4 9 7}$ & 1.0944 & 0.8500 & 0.9577 & 1.1834 & 0.9575 \\
8. I1 - COL & 0.8919 & 1.0690 & 0.8922 & 1.0063 & 1.2197 & 1.0058 \\
9. I2 - OL & 0.8601 & 0.9917 & 0.8607 & $\mathbf{0 . 9 3 7 5}$ & 1.0820 & 0.9378 \\
10. I2 - COL & 0.8944 & 0.9832 & 0.8949 & $\mathbf{0 . 9 4 6 0}$ & 1.0194 & 0.9463 \\
11. I12* & 1.4030 & 1.6330 & 1.3930 & 1.0336 & 1.0635 & 1.0316 \\
12. I22* & 1.4030 & 1.6330 & 1.3930 & 1.0336 & 1.0635 & 1.0316 \\
13. I13* & 1.0270 & 1.1068 & 1.0269 & 0.9908 & 1.1426 & 0.9904 \\
14. I23* & 1.0036 & 1.0308 & 1.0038 & 0.9463 & 1.0074 & 0.9466 \\
\hline
\end{tabular}

Table 2: Normalized Averages, $\left(\left|1-\gamma_{O L}\right|>0.05, \quad \gamma_{m i n}=0.1\right)$

\begin{tabular}{|l|c|c|c|c|c|c|}
\hline & \multicolumn{3}{|c|}{ LINE SEARCH } & \multicolumn{3}{c|}{ TrusT REGiON } \\
\cline { 2 - 7 } METHOD & Iterations & Functions & Fun.-Grad. & Iterations & Functions & Fun.-Grad. \\
\hline 0. BFGS & 1.0000 & 1.0000 & 1.0000 & 1.0000 & 1.0000 & 1.0000 \\
1. OL -BFGS & 1.2112 & 1.2071 & 1.2088 & 1.0961 & 1.1650 & 1.0959 \\
2. COL-BFGS & 1.1274 & 1.1746 & 1.1259 & 1.1440 & 1.2255 & 1.1423 \\
3. B1 - OL & 1.2679 & 1.2184 & 1.2671 & 1.1662 & 1.1671 & 1.1653 \\
4. B1 - COL & 1.1689 & 1.1364 & 1.16882 & 1.0831 & 1.0975 & 1.0825 \\
5. B2 - OL & 1.2833 & 1.2407 & 1.2823 & 1.1897 & 1.1878 & 1.1886 \\
6. B2 - COL & 1.1884 & 1.1543 & 1.1875 & 1.0821 & 1.0837 & 1.0815 \\
7. I1 - OL & 1.6885 & 2.0295 & 1.6853 & 1.4871 & 1.9763 & 1.4831 \\
8. I1 - COL & 1.1712 & 1.5095 & 1.1695 & 1.4075 & 1.8285 & 1.4048 \\
9. I2 - OL & 1.3490 & 1.4927 & 1.3462 & 1.2107 & 1.4682 & 1.2073 \\
10. I2 - COL & 1.1823 & 1.3410 & 1.1800 & 1.1997 & 1.4089 & 1.1967 \\
11. I12* & 1.2986 & 3.4839 & 1.2877 & 1.5460 & 2.1660 & 1.5425 \\
12. I22* & 1.2986 & 3.4839 & 1.2877 & 1.5460 & 2.1660 & 1.5425 \\
13. I13* & 0.9455 & 2.2305 & 0.9447 & 1.3927 & 2.0251 & 1.3907 \\
14. I23* & 0.9299 & 1.9855 & 0.9295 & 1.2172 & 1.4421 & 1.2177 \\
\hline
\end{tabular}


Each of the 14 procedures described in the Implementation Section 6.1 was used to solve the five problems mentioned above. Each problem was solved with the four initial matrix approximations listed in (6.3) using both line-search and trust-region globalization techniques. We use three measures for comparisons: number of iterations, number of function evaluations, and number of function-gradient evaluations (function evaluations plus $n$ times gradient evaluations, where $\mathrm{n}$ is the dimension of the problem). The results are presented as the average of each measure over the 20 outputs for each procedure. Our comparisons will be given relative to the control (standard BFGS). We accomplish this by listing all quantities normalized (divided) by the corresponding quantity for the BFGS control.

For each method and each globalization technique, the tables in this section present the average of the normalized data for each of our three measures. Tables 1 and 2 use $\bar{\gamma}=\gamma_{O L}$ in the Selective Sizing/Shifting Criteria 1 and 2, respectively. Similarly, Tables 3 and 4 use $\bar{\gamma}=\gamma_{C O L}$. All four tables use $\epsilon=0.05$ and $\gamma_{\min }=0.1 \mathrm{in}$ the selective sizing/shifting criteria as suggested by Contreras and Tapia[7].

From these results, we can see that in general:

1. The improvement for the line-search globalization technique is better than it is for the trust-region globalization technique, which indicates that trust region is a more robust technique, and therefore less sensitive to clianges such as selective sizing or shifting.

2. The Selective Sizing/Shifting Criterion 1 (Tables 1 and 3) is much better than Criterion 2 (Tables 2 and 4). This supports Contreras-Tapia's reason for sizing only when the sizing factor is less than one [7].

3. The number of iterations and function-gradient evaluations (which are similar) improved mucl more than the number of function evaluations. This further supports the explanation mentioned above because both globalization techniques are implemented to take care of "large" steps ("small" B's or $\bar{\gamma}>1$ ) at the expense of more than one function evaluation per iteration. Sizing only whien $\bar{\gamma}<1$ increases the number of "large" steps, which decreases the number of iterations, but it may increase the total number of function evaluations.

4. Using the identity matrix as a weighting matrix in the computation of the optimal values $\gamma^{*}$ or $\phi^{*}$ gives better results than using the matrix $B$. A plausible reason for this is that when $\bar{\gamma} \neq 1$, the matrix $B$ cloes not lave "good" information relative to $\nabla^{2} f(x)$. Therefore it is desirable to de-emphasize the role of $B$ and use a step that has a some "gradient" flavor (weighted matrix equal to the identity) to safeguard the BFGS method.

5. The optimal sizing factors given by Minimization Problem 2 in Sections 3 and 4 (Methods 11 and 12) always failed as a safeguard for the BFGS method. However, Minimization Problem 1 and 3 (Methods 3-10, 13 and 14) sometimes improved the behavior of the standard BFGS method, and slightly more than standard sizing techniques (Methods 1 and 2). This says that to safeguard the BFGS method, it is better to size another member of the Broyden class of secant updates than to just size the BFGS itself, i.e., when the BFGS is not performing well "move on" to 
Table 3: Normalized Averages, $\left(1-\gamma_{C O L}>0.05, \quad \gamma_{m i n}=0.1\right)$

\begin{tabular}{|l|c|c|c|c|c|c|}
\hline \multirow{2}{*}{ METHOD } & \multicolumn{3}{|c|}{ LINE SEARCH } & \multicolumn{3}{c|}{ TRUST REGION } \\
\cline { 2 - 7 } & Iterations & Functions & Fun.-Grad. & Iterations & Functions & Fun.-Grad. \\
\hline 0. BFGS & 1.0000 & 1.0000 & 1.0000 & 1.0000 & 1.0000 & 1.0000 \\
1. OL -BFGS & 0.9336 & 1.0110 & 0.9337 & 0.9706 & 1.0501 & 0.9710 \\
2. COL-BFGS & 0.8686 & 0.9613 & 0.8692 & 0.9430 & 1.0633 & 0.9429 \\
3. B1 - OL & 0.9851 & 1.0050 & 0.9853 & 0.9828 & 1.0087 & 0.9832 \\
4. B1 - COL & 0.9029 & 0.9651 & 0.9032 & 0.9651 & 1.0101 & 0.9654 \\
5. B2 - OL & 0.9326 & 0.9869 & 0.9328 & 0.9729 & 1.0083 & 0.9732 \\
6. B2 - COL & 0.8989 & 0.9619 & 0.8992 & 0.9713 & 1.0138 & 0.9717 \\
7. I1 - OL & 1.0802 & 1.3142 & 1.0796 & 1.0255 & 1.1725 & 1.0251 \\
8. I1 - COL & 0.8791 & 1.1248 & 0.8791 & 0.9837 & 1.1691 & 0.9836 \\
9. I2 - OL & 0.9895 & 1.1661 & 0.9887 & 1.0047 & 1.1365 & 1.0044 \\
10. I2 - COL & $\mathbf{0 . 8 5 2 4}$ & 1.0177 & 0.8556 & $\mathbf{0 . 9 4 4 2}$ & 1.0900 & 0.9463 \\
11. I12* & 1.4086 & 1.6368 & 1.3984 & 1.0454 & 1.0798 & 1.0431 \\
12. I22* & 1.4086 & 1.6368 & 1.3984 & 1.0454 & 1.0798 & 1.0431 \\
13. I13* & 1.0227 & 1.1233 & 1.0222 & 0.9864 & 1.1181 & 0.9861 \\
14. I23* & 1.0044 & 1.0307 & 1.0043 & 0.9854 & 1.0342 & 0.9854 \\
\hline
\end{tabular}

Table 4: Normalized Averages, $\left(\left|1-\gamma_{C O L}\right|>0.05, \quad \gamma_{\min }=0.1\right)$

\begin{tabular}{|l|c|c|c|c|c|c|}
\hline & \multicolumn{3}{|c|}{ LINE SEARCH } & \multicolumn{3}{c|}{ TRUsT REGION } \\
\cline { 2 - 7 } METHOD & Iterations & Functions & Fun.-Grad. & Iterations & Functions & Fun.-Grad. \\
\hline 0. BFGS & 1.0000 & 1.0000 & 1.0000 & 1.0000 & 1.0000 & 1.0000 \\
1. OL -BFGS & 1.2112 & 1.2071 & 1.2088 & 1.0961 & 1.1650 & 1.0959 \\
2. COL-BFGS & 1.1274 & 1.1746 & 1.1259 & 1.1440 & 1.2255 & 1.1423 \\
3. B1 - OL & 1.2747 & 1.2165 & 1.2740 & 1.1430 & 1.1212 & 1.1420 \\
4. B1 - COL & 1.1329 & 1.1056 & 1.1322 & 1.0978 & 1.0956 & 1.0970 \\
5. B2 - OL & 1.1704 & 1.1270 & 1.1696 & 1.1465 & 1.1388 & 1.1454 \\
6. B2 - COL & 1.1606 & 1.1315 & 1.1596 & 1.1228 & 1.1151 & 1.1220 \\
7. I1 - OL & 1.9117 & 2.3055 & 1.9075 & 1.5109 & 2.0212 & 1.5054 \\
8. I1 - COL & 1.2292 & 1.6407 & 1.2266 & 1.5080 & 1.9200 & 1.5027 \\
9. I2 - OL & 1.2907 & 1.4182 & 1.2876 & 1.2032 & 1.3705 & 1.1999 \\
10. I2 - COL & 1.1703 & 1.3073 & 1.1679 & 1.2347 & 1.4600 & 1.2307 \\
11. I12* & 1.3083 & 3.5063 & 1.2973 & 1.6134 & 2.3020 & 1.6086 \\
12. I22* & 1.3083 & 3.5063 & 1.2973 & 1.6134 & 2.3020 & 1.6086 \\
13. I13* & 0.9656 & 2.2585 & 0.9642 & 1.4929 & 2.2196 & 1.4886 \\
14. I23* & 0.9481 & 2.0051 & 0.9471 & 1.2705 & 1.5207 & 1.2690 \\
\hline
\end{tabular}


Table 5: Normalized Averages, $\left(1-\gamma_{C O L}>0.1, \gamma_{\min }=0.1\right)$

\begin{tabular}{|l|c|c|c|c|c|c|}
\hline & \multicolumn{3}{|c|}{ LINE SEARCH } & \multicolumn{3}{c|}{ TRUST REGION } \\
\cline { 2 - 7 } METHOD & Iterations & Functions & Fun.-Grad. & Iterations & Functions & Fun.-Grad. \\
\hline 0. BFGS & 1.0000 & 1.0000 & 1.0000 & 1.0000 & 1.0000 & 1.0000 \\
1. OL -BFGS & 0.9303 & 1.0077 & 0.9305 & 0.9834 & 1.0682 & 0.9837 \\
2. COL-BFGS & 0.8745 & 0.9678 & 0.8747 & 0.9776 & 1.0473 & 0.9775 \\
3. B1 - OL & 0.9369 & 0.9917 & 0.9371 & 0.9700 & 1.0190 & 0.9703 \\
4. B1 - COL & 0.9059 & 0.9686 & 0.9062 & 0.9577 & 1.0108 & 0.9580 \\
5. B2 - OL & 0.9279 & 0.9891 & 0.9281 & 0.9666 & 0.9971 & 0.9669 \\
6. B2 - COL & 0.9027 & 0.9672 & 0.9030 & 0.9646 & 1.0203 & 0.9649 \\
7. I1 - OL & 0.9920 & 1.1871 & 0.9915 & 1.0113 & 1.1796 & 1.0109 \\
8. I1 - COL & 0.8732 & 1.1066 & 0.8733 & 0.9670 & 1.1475 & 0.9669 \\
9. I2 - OL & 0.9746 & 1.1410 & 0.9738 & 1.0090 & 1.1335 & 1.0087 \\
10. I2 - COL & $\mathbf{0 . 8 5 4 0}$ & 1.0057 & 0.8543 & $\mathbf{0 . 9 4 5 6}$ & 1.0897 & 0.9457 \\
11. I12* & 1.2608 & 1.6543 & 1.2508 & 1.0602 & 1.2437 & 1.0577 \\
12. I22* & 1.2608 & 1.6543 & 1.2508 & 1.0602 & 1.2437 & 1.0577 \\
13. I13* & 0.8634 & 1.0813 & 0.8634 & 0.9347 & 1.1377 & 0.9349 \\
14. I23* & 0.8549 & 1.0034 & 0.8552 & 0.9605 & 1.0601 & 0.9608 \\
\hline
\end{tabular}

another update instead of trying to patch up the BFGS. A similar conclusion was reached by $\mathrm{Al}$-Baali[2] in his numerical experimentation using his switching methods.

6. The best results are obtained when there is a direct correlation between $\bar{\gamma}$, the quantity used to decide when to size or shift, and $\gamma$, the sizing factor actually used. When $\bar{\gamma}=\gamma_{O L}$, Methods 7 and 9, which used $\gamma=\gamma_{O L}$ as the sizing factor, saved $15 \%$ and $6.3 \%$ over BFGS with line searcl and trust region, respectively (Table 1 ). Similarly, when $\bar{\gamma}=\gamma_{C O L}$, the best method, I2-COL, used $\gamma_{C O L}$ as the sizing factor and saved $14.5 \%$ and $5.4 \%$ (Table 3). Notice that the same is true not only for the choices that gave the best results.

7. Notice that the results given ahove are slightly better than those for Method 2, which was the choices considered by Contreras and Tapia[7] (see Tables 1 and 3).

\subsection{More Results}

Further numerical experimentation was done to determine the sensitivity of our results to both the dimension of the problems and choices of $\epsilon$ and $\gamma_{m i n}$. For the second analysis, we computed the same type of information as the tables above for different values of $\epsilon$ and $\gamma_{\min }$ using Selective Sizing/Shifting Criterion 1. Tables 5 and 6 show this information for $\gamma=\gamma_{C O L}$ and $\left(\epsilon, \gamma_{\min }\right)$ equal to $(0.1,0.1)$ and $(0.05,0.001)$, respectively. These are typical results for this sensitivity analysis.

From this data, we can see that the results obtained from the standard sizing procedures (Methods 1 and 2) became worse as $\epsilon$ increased or $\gamma_{m i n}$ decreased, while the other 
Table 6: Normalized Averages, $\left(1-\gamma_{C O L}>0.05, \quad \gamma_{\min }=0.001\right)$

\begin{tabular}{|l|c|c|c|c|c|c|}
\hline & \multicolumn{3}{|c|}{ LINE SEARCH } & \multicolumn{3}{c|}{ ThuST REGION } \\
\cline { 2 - 7 } METHOD & Iterations & Functions & Fun.-Grad. & Iterations & Functions & Fun.-Grad. \\
\hline 0. BFGS & 1.0000 & 1.0000 & 1.0000 & 1.0000 & 1.0000 & 1.0000 \\
1. OL -BFGS & 0.9856 & 1.0245 & 0.9857 & 1.0100 & 1.0670 & 1.0103 \\
2. COL-BFGS & 0.8714 & 0.9584 & 0.8716 & 0.9728 & 1.0542 & 0.9727 \\
3. B1 - OL & 0.9387 & 0.9859 & 0.9388 & 0.9731 & 1.0167 & 0.9734 \\
4. B1 - COL & 0.9067 & 0.9671 & 0.9070 & 0.9651 & 1.0101 & 0.9654 \\
5. B2 - OL & 0.9380 & 0.9920 & 0.9382 & 0.9729 & 1.0083 & 0.9732 \\
6. B2 - COL & 0.9075 & 0.9659 & 0.9078 & 0.9713 & 1.0138 & 0.9717 \\
7. I1 - OL & 1.0337 & 1.2743 & 1.0331 & 1.0255 & 1.1725 & 1.0251 \\
8. I1 - COL & 0.8849 & 1.1249 & 0.8848 & 0.9837 & 1.1691 & 0.9836 \\
9. I2 - OL & 1.0352 & 1.2263 & 1.0340 & 1.0047 & 1.1365 & 1.0044 \\
10. I2 - COL & 0.8721 & 1.0278 & 0.8722 & 0.9462 & 1.0900 & 0.9463 \\
11. I12* & 1.2284 & 1.9570 & 1.2184 & 1.1360 & 1.3849 & 1.1332 \\
12. I22* & 1.2284 & 1.9570 & 1.2184 & 1.1360 & 1.3849 & 1.1332 \\
13. I13* & 0.8648 & 1.3652 & 0.8647 & 0.9857 & 1.1960 & 0.9857 \\
14. I23* & 0.8523 & 1.2269 & 0.8525 & 0.9960 & 1.1475 & 0.9961 \\
\hline
\end{tabular}

procedures remained mostly unchanged (with exception of Methods 13 and 14, which improved with these changes). Of course, this holds within some ranges of $\epsilon$ and $\gamma_{\min }$. In fact, with $\epsilon>.2$, the normalized data is close to one, ${ }^{8}$ and it is greater than one when $\gamma_{\min }$ goes to zero. ${ }^{9}$

To check the sensitivity of our results to the climension of the problems, we ran our code for problem number 21 with several values of $n$ between 10 and 30 , and the starting points and initial matrices used by Contreras and Tapia[7]. Again, we used the Selective Sizing/Shifting Criterion 1 with $\bar{\gamma}=\gamma_{C O L}$ and $\gamma_{m i n}=0.1$.

Since now the issue is reliability rather than efficiency, Table 7 presents, for $n=20$, the number of iterations used by each procedure to converge for the different starting points and initial matrices used in trust-region sized BFGS methods. Here, the starting points are

$$
\begin{aligned}
x_{0}^{1} & =(-1.2,1, \ldots,-1.2,1) \\
x_{0}^{2} & =(-4000,1,-1.2,1, \ldots,-4000,1,-1.2,1) \\
x_{0}^{3} & =(-100.5,40, \ldots,-100.5,40) \\
x_{0}^{4} & =(-40,20,20,20, \ldots,-40,20,20,20) \\
x_{0}^{5} & =(-300,-100, \ldots,-300,-100)
\end{aligned}
$$

\footnotetext{
${ }^{8}$ This means that if $\epsilon$ is not small enough, there is not enough sizing to really safeguard (improve) the behavior of the standard BFGS method.

${ }^{9}$ This shows the importance of preventing the sizing strategies from using too small sizing factors.
} 
Table 7: Number of Iterations. (Problem 21 with $n=20,1-\gamma_{C O L}>0.05, \quad \gamma_{\min }=0.1$ )

\begin{tabular}{|c|c|c|c|c|c|c|c|c|c|c|c|c|c|c|c|c|}
\hline \multirow{2}{*}{\multicolumn{2}{|c|}{$\begin{array}{l}\text { INITIAL } \\
\text { VALUES }\end{array}$}} & \multicolumn{15}{|c|}{$\mathrm{METHODS}$} \\
\hline & & \multicolumn{3}{|c|}{ BFGS } & \multicolumn{2}{|c|}{$\mathrm{B} 1$} & \multicolumn{2}{|c|}{ I1 } & \multirow[b]{2}{*}{$\mathrm{I} 12^{*}$} & \multirow[b]{2}{*}{$113^{*}$} & \multicolumn{2}{|c|}{$\overline{\mathrm{B} 2}$} & \multicolumn{2}{|c|}{$\mathrm{I} 2$} & \multirow[b]{2}{*}{ I $22^{*}$} & \multirow[b]{2}{*}{$\mathrm{I} 23^{*}$} \\
\hline$x_{0}$ & $B_{0}$ & Stand & $\mathrm{OL}$ & $\mathrm{COL}$ & $\mathrm{OL}$ & $\mathrm{COL}$ & $\mathrm{OL}$ & $\mathrm{COL}$ & & & $\mathrm{OL}$ & $\mathrm{COL}$ & $\mathrm{OL}$ & $\mathrm{COL}$ & & \\
\hline \multirow[t]{4}{*}{$x_{0}^{1}$} & $B_{0}^{1}$ & 138 & 147 & 140 & 149 & 142 & 144 & 139 & 142 & 138 & 152 & 147 & 146 & 137 & 142 & 169 \\
\hline & $B_{0}^{2}$ & $\mathrm{~F}$ & 197 & 149 & 167 & 146 & $\mathrm{G}^{* *}$ & 152 & $\mathrm{G}^{* *}$ & 143 & 157 & 143 & 197 & 147 & $\mathrm{G}^{* *}$ & $\mathrm{G}^{* *}$ \\
\hline & $B_{0}^{3}$ & 183 & 375 & 360 & 286 & 384 & 206 & 292 & $\mathrm{~F}^{*}$ & 356 & 219 & 394 & 196 & 313 & $\mathrm{~F}^{*}$ & 348 \\
\hline & $B_{0}^{4}$ & 206 & 279 & 382 & 294 & 345 & 219 & 293 & 407 & 350 & 301 & 334 & 212 & 297 & 407 & 346 \\
\hline \multirow[t]{4}{*}{$x_{0}^{2}$} & $B_{0}^{1}$ & $\mathrm{~F}$ & $\mathrm{~F}$ & $\bar{F}$ & $\mathrm{~F}$ & $\mathrm{~F}$ & $\mathrm{~F}$ & $\bar{F}$ & $\mathrm{~F}$ & $F$ & $\mathrm{~F}$ & $\bar{F}$ & $\mathrm{~F}$ & $\bar{F}$ & $\bar{F}$ & $F$ \\
\hline & $B_{0}^{2}$ & $\mathrm{~F}$ & 284 & 253 & 204 & 221 & 425 & 298 & $\mathrm{~F}$ & $\mathrm{~F}$ & 213 & 218 & 284 & 294 & $\mathrm{~F}$ & $\mathrm{~F}$ \\
\hline & $B_{0}^{3}$ & $\mathrm{~F}$ & $\mathrm{~F}$ & $\mathrm{~F}$ & $\mathrm{~F}$ & $\mathrm{~F}$ & $\mathrm{~F}$ & $\mathrm{~F}$ & $\mathrm{~F}$ & $\mathrm{~F}$ & $\mathrm{~F}$ & $\mathrm{~F}$ & $\mathrm{~F}$ & $\mathrm{~F}$ & $\mathrm{~F}$ & $F$ \\
\hline & $B_{0}^{4}$ & $F$ & $\mathrm{~F}$ & $\mathrm{~F}$ & $\mathrm{~F}$ & $\mathrm{~F}$ & $\mathrm{~F}$ & $\mathrm{~F}$ & $\mathrm{~F}$ & $\mathrm{~F}$ & $\mathrm{~F}$ & $\mathrm{~F}$ & $\mathrm{~F}$ & $\mathrm{~F}$ & $\mathrm{~F}$ & $F$ \\
\hline \multirow[t]{4}{*}{$x_{0}^{3}$} & $\bar{B}_{0}^{1}$ & $\bar{F}$ & 472 & 487 & $\mathrm{~F}^{* *}$ & 464 & 498 & 413 & $\bar{F}$ & $\mathrm{~F}^{*}$ & $\mathrm{~F}^{* *}$ & 486 & 472 & 487 & $\bar{F}$ & $\mathrm{~F}^{* *}$ \\
\hline & $B_{0}^{2}$ & $\mathrm{~F}$ & 372 & 393 & 417 & 344 & 446 & 373 & 280 & $\mathrm{G}^{* *}$ & 392 & 342 & 442 & 364 & 280 & 295 \\
\hline & $B_{0}^{3}$ & $\mathrm{~F}$ & $\mathrm{~F}$ & $\mathrm{~F}$ & $\mathrm{~F}^{*}$ & $\mathrm{~F}^{*}$ & $\mathrm{~F}$ & $\mathrm{~F}^{* *}$ & $\mathrm{~F}$ & $\mathrm{~F}$ & $\mathrm{~F}$ & $\mathrm{~F}^{*}$ & $\mathrm{~F}$ & $\mathrm{~F}^{*}$ & $\mathrm{~F}$ & $\mathrm{~F}$ \\
\hline & $B_{0}^{4}$ & $\mathrm{~F}$ & $\mathrm{~F}$ & $\mathrm{~F}^{*}$ & 463 & 447 & $\mathrm{~F}^{*}$ & 465 & 489 & $\mathrm{~F}$ & 472 & 423 & 498 & 484 & 489 & $\mathrm{~F}$ \\
\hline \multirow[t]{4}{*}{$x_{0}^{4}$} & $B_{0}^{1}$ & 334 & 258 & 263 & 267 & 259 & 264 & 263 & 247 & 238 & 251 & 255 & 257 & 261 & 247 & 250 \\
\hline & $B_{0}^{2}$ & $\mathrm{~F}$ & 367 & 291 & 319 & 268 & 367 & 310 & 221 & 216 & 319 & 255 & 367 & 291 & 221 & 221 \\
\hline & $B_{0}^{3}$ & 416 & $\mathrm{~F}$ & $\mathrm{~F}$ & 359 & 426 & 335 & 446 & $F^{*}$ & $\mathrm{~F}^{*}$ & 391 & 443 & 389 & 430 & $\mathrm{~F}^{*}$ & $\mathrm{~F}$ \\
\hline & $B_{0}^{4}$ & 372 & 453 & 440 & 314 & 317 & 332 & 328 & 339 & 338 & 299 & 339 & 325 & 317 & 339 & 333 \\
\hline \multirow[t]{4}{*}{$x_{0}^{5}$} & $B_{0}^{1}$ & $\mathrm{~F}^{*}$ & 430 & 390 & 397 & 365 & 428 & 390 & 330 & 348 & $\overline{382}$ & 363 & 430 & 390 & 330 & 369 \\
\hline & $B_{0}^{2}$ & $\mathrm{~F}$ & 414 & 274 & 303 & 245 & 231 & 343 & $\mathrm{~F}$ & $\mathrm{~F}$ & 331 & 245 & 237 & 346 & $\mathrm{~F}$ & $F$ \\
\hline & $B_{0}^{3}$ & $\mathrm{~F}$ & $\mathrm{~F}$ & $\mathrm{~F}$ & $\mathrm{~F}$ & $\mathrm{~F}$ & $\mathrm{~F}$ & $\mathrm{~F}$ & $\mathrm{~F}$ & $\mathrm{~F}$ & $\mathrm{~F}$ & $\mathrm{~F}$ & $\mathrm{~F}$ & $\mathrm{~F}$ & $F$ & $\mathrm{~F}$ \\
\hline & $B_{0}^{4}$ & $\mathrm{~F}^{*}$ & $\mathrm{~F}$ & $\mathrm{~F}$ & 413 & 386 & 381 & 377 & 375 & 385 & 407 & 390 & 393 & 372 & 375 & 384 \\
\hline
\end{tabular}

F: Maximum number of iterations (500) reached.

G: Global strategy failed to compute a "goor" step.

*: Last iterate was "close" to the solution (crror $\left.<10^{-2}\right)$.

**: Last iterate was "very close" to the solution (error $\left.<10^{-5}\right)$. 
and the initial matrices are

$$
\begin{aligned}
& B_{0}^{1}=I \\
& B_{0}^{2}=I+\left(10^{12}-1\right) * \operatorname{diag}\left(1-\frac{\mathrm{i}-1}{\mathrm{n}-1}\right) \\
& B_{0}^{3}=\operatorname{diag}\left(10^{7}, 10^{-7}, \ldots, 10^{7}, 10^{-7}\right), \\
& B_{0}^{4}=\operatorname{diag}\left(10^{5}, 10^{-5}, \ldots, 10^{5}, 10^{-5}\right) .
\end{aligned}
$$

From these results, we can see that an appropriate sizing procedure is needed to improve the reliability of the standard BFGS method, as was also concluded by Contreras and Tapia[7]. Even though none of the 14 procedures tested lere were $100 \%$ reliable, the most reliable procedures were those derived from Minimization Problem 1 in Sections 3 and 4 (columns 6-9 and 12-15 in Table 7). This fact further supports Observation 5 made above, which says that BFGS should be safeguarding by sizing another update instead of patching up the BFGS.

Observe also that, ignoring the cases for which all procedures failed, ${ }^{10}$ these most reliable procedures were "close" or "very close" to the solution in the cases they failed, and in these cases, the standard BFGS completely failed. It should be noticed that this was not true for Method 2, the Contreras-Tapia choice (see case $x_{0}^{4}$ with $B_{0}^{3}$ ).

\section{Summary and Concluding Remarks}

In this study we have presented considerable theoretical and numerical discussion concerning sizing members of the Broyden family of secant updates. This study has led us to the following conclusions. The BFGS secant update should continue to be the secant method of choice for unconstrained optimization. However, the BFGS update, on occasion, produces a faulty update. This occurrence can be detected using either the Oren-Luenberger sizing factor (1.10) or the centered Oren-Luenberger sizing factor (1.11). When such a situation is detected the approximate Hessian should be sized with the sizing factor used to detect this anomaly. These findings mirror those of Contreras and Tapia [7]. However, we have discovered more.

The BFGS update is prone to producing updates with large eigenvalues. Indeed, in some way it was designed to do this, since it was presented as a fix for the DFP method which produced excessively small eigenvalues: Hence sizing the BFGS update may lead to an excessively small sizing factor and near singularity of the sized matrix. Contreras and Tapia were aware of this deficiency and suggested a safeguard for their sizing strategy. Another way to compensate for this negative feature is to shift, i.e., add a matrix to the sized matrix. The effective implementation of this shift has been the primary subject of the present study. We first considered a two-parameter family consisting of sized Broyden family secant updates. It was instructive for us to view this two-parameter family as a BFGS update of a matrix $\gamma B+\phi v v^{T}$ which had been sized and then shifted. The shift is of the form $\phi v v^{T}$ and corresponds to switching from the BFGS update $(\phi=0)$ to an alternative member of the Broyden family of updates $(\phi \neq 0)$. Considerable theory and validating numerical experimentation was performed in an effort to find good choices for

\footnotetext{
${ }^{10}$ In one of these cases, $x_{0}^{3}$ with $B_{0}^{3}$, Proceclure I1-COL was the only one that almost solved the problem.
} 
the sizing parameter $\gamma$ and the shifting parameter $\phi$. After much study we concluded (as mentioned above) that the sizing factor should be either the Oren-Luenberger or the centered-Oren-Luenberger sizing factor and the sizing should be done selectively as suggested by Contreras and Tapia [7]. Our numerical experimentation indicated that an effective way of determining the slift factor $\phi$ was to minimize the Byrd-Nocedal $\psi$-measure over our sized Broyden family with the sizing factor fixed. We considered weighting the Byrd-Nocedal $\psi$-measure by a matrix $D$ and studied the following three choices for $D: D=I$ the identity, $D=B$ the approximate Hessian, and $D=\nabla^{2} f(x)$ the exact Hessian. The choice $D=I$ gives the member of the sized Broyden family which is nearest the method of steepest descent (in the Byrd-Nocedal measure), the choice $D=B$ gives the least-change member, and the choice $D=\nabla^{2} f(x)$ gives the member which is nearest to Newton's method. We therefore were rather surprised to find that our numerically most effective choice was $D=I$. In retrospect we see that there is considerable consistency in this choice. Our sizing indicator has told us that we should size; hence there is a bad match of information in the pair $B$ and $\nabla^{2} f(x)$. The choice $D=I$ prevents this faulty information from further contaminating our update.

Acknowledgements. The authors thank Martha Contreras and Mónica Martínez for assistance in some of the early experimentation. 


\section{References}

[1] M.Al-Baali (1988), Partial self scaling variable metric algorithms, Report N80, Dipartimento di Sistemi, Universita della Calabria, Italy, October.

[2] M.Al-Baali (1992), An efficient class of quasi-Newton algorithms in the Broyden family, Technical Report N.9, Dipartimento di Elettronica Informatica e Sistemistica, Universita della Calabria, Italy, March.

[3] R.H.Byrd, J.Nocedal and Y.X.Yuan (1987), Global convergence of a class of quasiNewton methods on convex problems SIAM J. Numerical Analysis, 24, 1171-1190.

[4] R.H.Byrd and J.Nocedal (1989), A tool for the analysis of quasi-Newton methods with application to unconstrained minimization, SIAM J. Numerical Analysis, 26, 727-739.

[5] R.H.Byrd, D.C.Liu and J.Nocedal (1990), On the behavior of Broyden's class of quasiNewton methods, Technical Report NAM 01, Department of Electrical Engineering and Computer Science, Northwestern University, Evanston, Illinois, USA, May.

[6] R.G.Carter (1987) Safeguarding Hessian approximations in trust region algorithms. Report TR87-12, Department of Mathematical Sciences, Rice University, Texas, USA, June.

[7] M.Contreras and R.A.Tapia (1991), Sizing the BFGS and DFP updates: A numerical study, JOTA, 78, 93-108.

[8] W.C.Davidon (1975), Optimally conditioned optimization algorithms without line searches, Mathematical Programming, 9, 1-30.

[9] J.E.Dennis Jr. and R.B.Schnabel (1983), Numerical Methods for Unconstrained Optimization and Nonlinear Equations, Prentice-Hall, New Jersey.

[10] J.E.Dennis Jr. and H.Wolkowicz (1990), Sizing and least change secant methods, Report TR90-5, Department of Mathematical Sciences, Rice University, Texas, USA, March.

[11] J.E.Dennis Jr., D.M.Gay and R.E.Welsch (1981), An adaptive nonlinear least-squares algorithm, ACM Transactions on Mathematical Softuare, 7, 348-368.

[12] R.Fletcher (1991), A new variational result for quasi-Newton formulae, SIAM J. Optimization, 1, 18-21.

[13] L.Luksan (1990), Computational experience with inproved variable metric Methods for unconstrained minimization, Kybernetika, 26, 415-431.

[14] J.J.Moré, B.S.Garbow and K.E.Hillstrom (1981), Testing unconstrained optimization software, ACM Transactions on Mathematical Software, 7, 17-41. 
[15] J.Nocedal and Y.Yuan (1991), Analysis of a self-scaling quasi-Newton method, Technical Report NAM-02, Department of Electrical Engineering and Computer Science, Northwestern University, Evanston, Illinois, September.

[16] S.S.Oren (1974), Self-scaling variable metric (SSVM) algorithms, Part 2: Implementation and experiments, Management Science, 20, 863-874.

[17] S.S.Oren and D.G.Luenberger (1974), Self-scaling variable metric (SSVM) algorithms, Part 1: Criteria and sufficient conditions for scaling a class of algorithms, Management Science, 20, 845-862.

[18] S.S.Oren and E.Spedicato (1976), Optimal conditioning of self-scaling variable metric algorithm, Mathematical Programming, 10, 70-90.

[19] J.D.Pearson (1969), Variable metric methods of minimization, Computer J., 12, 171178.

[20] R.B.Schnabel (1978), Optimal conditioning in the convex class of rank two updates, Mathematical Programming, 15, 247-260.

[21] D.F.Shanno and K.Phua (1978), Matrix conditioning and nonlinear optimization, Mathematical Programming, 14, 149-16i0.

[22] M.J.Todd (1991), Another variational derivation of a self-scaling quasi-Newton update formula, Technical Report, No.964, School of Operations Research and Industrial Engineering, College of Engineering, Cornell University, Ithaca, New York, April.

[23] H.Wolkowicz (1991), Measures for symmetric rank-one updates, Report, Department of Combinatorics and Optimization, University of Waterloo, Ontario, Canada, October.

[24] H.Yabe and N.Yamaki (1980), Some properties of Oren's SSVM type algorithm for unconstrained minimization, TRU Mathemutics, 16, No.2, 103-111.

[25] Y.Zhang and R.P.Tewarson (1988), Quasi-Newton algorithms with updates from the preconvex part of Broyden's family, IMA Journal of Numerical Analysis, 8, 487-509. 